\title{
Armes reiches Land
}

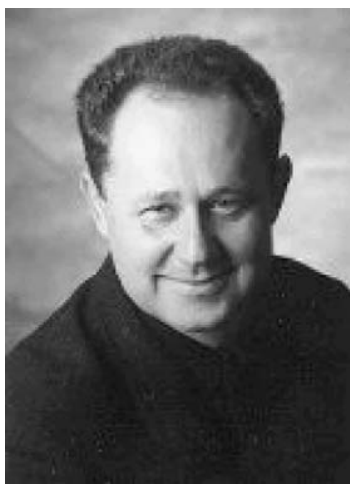

Prof. Dr. med. Manfred Wildner

Bibliografie

DOI http://dx.doi.org/

10.1055/s-0033-1333776

Gesundheitswesen 2013;

75: 65-66

(c) Georg Thieme Verlag KG

Stuttgart · New York

ISSN 0941-3790

Korrespondenzadresse

Prof. Dr. med. Manfred Wildner

Bayerisches Landesamt für Gesundheit und Lebensmittelsicherheit

Veterinärstraße 2

85764 Oberschleißheim

manfred.wildner@|gl.bayern.de
Die Kinder- und Hausmärchen der Brüder Jacob und Wilhelm Grimm (Erstauflage 1812) sind nach der Lutherbibel das wohl am weitesten verbreitete literarische Werk deutscher Sprache. Als Gründungsväter der Germanistik und darüber hinaus der indoeuropäischen Sprachforschung allgemein anerkannt, ist der politische Einsatz der Brüder Grimm für eine freiheitliche Verfassung auf deutschem Boden weniger bekannt: Als Mitglied der "Göttinger 7“ verloren sie wegen ihres Protestes gegen die Aufhebung der Hannoveraner Verfassung zeitweilig ihre Professorenämter - dem des Landes verwiesenen Jacob Grimm wurde das Gehalt aus Spenden der sich solidarisierenden Bevölkerung weiterbezahlt. Auffällig ist, dass ihre Märchen nach der fast schon obligatorischen Hochzeit des Königssohns oder der Königstocher mit dem lapidaren Halbsatz enden: „Und sie lebten vergnügt bis an ihr Ende“. Da es sich bei dem Zeitraum von der Hochzeit bis zum Tod zumeist um den größeren Lebensabschnitt handelt, kann zu Recht nachgefragt werden, ob hier nicht etwas fehlt. Vielleicht nicht für Kinderohren, aber wohl doch für die erwachsenen aufmerksamen Zuhörerinnen und Zuhörer.

Lässt sich gelungenes bzw. glückliches Leben, nicht nur für Angehörige der Oberschicht („Königstöchter und -söhne“), sondern auch der bürgerlichen Mittelschicht oder der unteren sozialen Schicht näher beschreiben? Eine mögliche Herangehensweise an diese Fragestellung geht auf Abraham Maslow zurück (1908-1970). Dem Gründervater der humanistischen Psychologie war zwar selbst kein langes Leben beschieden, jedoch haben seine Gedanken zur Motivation und Persönlichkeitsentwicklung weite Verbreitung gefunden [1]. Die sogenannte „Maslowsche Bedürfnispyramide" skizziert eine Abfolge von Lebensbedürfnissen, welche in hierarchischer Ordnung die Grund- und Existenzbedürfnisse wie Essen und Trinken, die Bedürfnisse nach Sicherheit und sozialer Eingebundenheit, die Individualbedürfnisse wie Anerkennung und Wertschätzung und als fünfte Stufe die Selbstverwirklichung umfassen. Oft übersehen wird, dass Maslow in seinem Todesjahr noch weitere Stufen ergänzt hat, insbesondere als neue Spitze die Befähigung zur Transzendenz: der Suche nach einer die Grenzen des individuellen Selbst übersteigenden Dimension [2]. Denkbar ist, dass die Königssöhne und Königstöchter aus den Märchen in ihren verschwiegenen späteren Biografien diese Bedürfnishierarchie erfolgreich durchlaufen haben, auch wenn nach einer Schätzung Maslows nur etwa $2 \%$ der Bevölkerung den Anspruch der
Selbstverwirklichung für sich zufriedenstellend lösen. Wahrscheinlich ist, dass die Mehrzahl der Menschen zumindest zu Zeiten der Brüder Grimm überwiegend mit der Sicherung der Grund- und Existenzbedürfnisse beschäftigt war. Was sind die Erkenntnisse über Armut und Reichtum im Deutschland des 21. Jahrhunderts? Für die Zwecke der offiziellen europäischen Statistiken wird zur Definition der Armutsgrenze Bezug zum mittleren Nettoeinkommen genommen („Median des Netto-Äquivalenz-Einkommens"). Beträgt dieses weniger als $70 \%$ des Durchschnitts, wird von einer Armutsgefährdung in sozialen Risikosituationen gesprochen, bei weniger als $60 \%$ von einer Armutsgefährdung, bei weniger als $50 \%$ von einer relativen Einkommensarmut und bei weniger als $40 \%$ von Armut. Weltgesundheitsorganisation und OECD definieren dem gegenüber Armut bereits ab einer Einkommensgrenze von 50\%.

Nach den Erkenntnissen des Armuts- und Reichtumsberichts der Bundesregierung, welcher unter dem Titel „Lebenslagen in Deutschland“ seit 2001 in regelmäßigen Abständen erscheint, wird von einer Armutsgefährdung bei etwa jedem achten Menschen in Deutschland berichtet. Dabei lohnt sich noch einmal eine genauere Beschäftigung mit dem Begriff der Armut, welcher bei einer Fokussierung auf das verfügbare Einkommen alleine zu kurz greift. In einer Definition der europäischen Gemeinschaft von 1984 gelten „Personen, Familien und Gruppen als arm [...], die über so geringe (materielle, kulturelle und soziale) Mittel verfügen, dass sie von der Lebensweise ausgeschlossen sind, die in dem Mitgliedsstaat, in dem sie leben, als Minimum annehmbar ist" [3]. Das ist der sog. „Lebenslagenansatz“ der Armutsforschung, wie er auch im Titel des Armuts- und Reichtumsberichts der Bundesregierung angesprochen wird. Prekäre Lebenslagen, etwa geringe Schulbildung, Arbeitslosigkeit oder alleinerziehend zu sein, stehen dabei naheliegender weise wiederum in engem Zusammenhang mit der einkommensbezogenen Armut. Während das Risiko für Paare mit steigender Kinderzahl ansteigt, liegt die Armutsrisikoquote für Alleinerziehende etwa doppelt so hoch wie in der Allgemeinbevölkerung: fast jeder Vierte bzw. jede Vierte ist davon betroffen. Und damit gilt dieses Armutsrisiko auch für etwa jedes fünfte Kind unter 15 Jahren in Deutschland.

Und da es ja auch ein Reichtumsbericht ist: Wie steht es um die modernen Königskinder? Der nach dem italienischen Statistiker Corrado Gini benannte Gini-Koeffizient als Maß der Einkom- 
mensungleichheit ist in Deutschland über die Jahre relativ konstant, wobei das private Vermögen recht ungleich verteilt ist, dem gegenüber mit zunehmender Tendenz. Ein Zehntel der in Deutschland lebenden Menschen gehört zu einer privilegierten Gruppe, welche über die Hälfte des Privatvermögens ihr Eigen nennt. Das mit dem Durchschnitt ist eben so eine Sache: „Am Durchschnitt ist die Kuh ersoffen“ sagt ein Bonmot und verweist dabei auf die Geschichte einer Kuh, welche beim Durchqueren einer an sich flachen Furt an einem tiefen Loch ums Leben kam. Solche „Löcher“ sind real und der Umgang mit Armut kennzeichnet ebenso wie der Umgang mit Reichtum das kulturelle Niveau einer Gesellschaft.

In Analogie zu den Gefahren einer Flussdurchquerung sind auch im Verlauf des Lebens eine Reihe von Übergängen zwischen Lebensphasen zu bestehen. Der Armuts- und Reichtumsbericht nennt hier nach der Geburt, unserer ersten Übergangsphase, die Inanspruchnahme frühkindlicher Förderung, den Schuleintritt und den Schulformwechsel, eine erfolgreiche Berufsausbildung, den Berufseinstieg und den eventuellen Wiedereinstieg nach Familiengründung, das Überstehen von Arbeitslosigkeit und der Erwerb einer weiteren beruflichen Qualifikation, den Eintritt in die Posterwerbsphase sowie den Eintritt von Behinderungen und Pflegebedürftigkeit. Hier leistet die gesellschaftliche Solidargemeinschaft in Deutschland vergleichsweise viel: Durch den Zugang zu Kindertagesbetreuungen, zu Schulen, Ausbildungsplätzen und Hochschulen, durch die Abfederung von Lebensrisiken durch verpflichtende Sozialversicherungssysteme, nicht zuletzt im Gesundheitsbereich und mit der Pflegeversicherung als fünfter Säule der Sozialversicherung, nach Krankenversicherung, Unfallversicherung, Rentenversicherung und Arbeitslosenversicherung.

Und wie steht es um den Zusammenhang von Armut bzw. Reichtum mit Gesundheit? Gute Gesundheit genießt nur etwa jeder bzw. jede Dritte mit niedriger Schulbildung gegenüber jedem bzw. jeder Zweiten mit mittlerer oder hoher Schulbildung. Auch wenn in der Wissenschaft noch darüber diskutiert wird, wie groß der Einfluss von Einkommen und Bildung auf den Gesundheitszustand sind, wie dieser Einfluss vermittelt wird und wie ein sozioökonomischer Abstieg infolge von Krankheit und Behinderung zu bewerten ist (Deprivations-Hypothese, SelektionsHypothese), ist die Existenz derartiger Zusammenhänge unstrittig [4]. Dabei hat neben der persönlich gestaltbaren Situation auch die umgebende Lebenswelt, physikalisch wie sozioökonomisch, einen großen Einfluss. Bei einer differenzierten Betrachtung sind auch der relative Wohlstand der Wohnumgebung, auch die relative eigene Position innerhalb des sozialen Umfeldes und auch die Aspekte der Umweltgerechtigkeit, d.h. der Exposition gegenüber schädigenden oder fördernden Einflüssen der physikalisch-chemischen Umwelt bedeutsam.

Der englische Epidemiologe und Sozialwissenschaftler Richard Wilkinson formulierte die Hypothese, dass - ab einem bestimmten Entwicklungsstand - weniger der absolute Grad an materiellem Reichtum oder materieller Armut ausschlaggebend für den Gesundheitszustand einer Bevölkerung sind, sondern die Ungleichheit in der Verteilung dieser materiellen Ressourcen [5]. Demnach wäre bei uns weniger das absolute Einkommen zu betrachten, sondern mehr die soziale und materielle Gerechtigkeit, Sozialklima und Sozialverhalten. Ein armes und gleichzeitig reiches Land ist nicht nur denkbar, sondern in vielen Gesellschaften Realität. Illustrieren lässt sich das z.B. durch die Lebenserwartung in Großstädten, die abhängig vom sozialen Umfeld bei jeder Haltestelle an gewissen U-Bahn-Linien spürbar und messbar fallen kann. Körperliche und psychische Gesundheit, Suchtverhalten, Bildungserfolg, Gefängnisstrafen, Übergewicht, sozialer Auf- und Abstieg, soziales Vertrauen, Gewalt, Teenage-Schwangerschaften und das Wohlergehen von Kindern weisen alle schlechtere Werte in Gesellschaften auf, in welchen der relative Reichtum sehr ungleich verteilt ist [6]. Und diese Beobachtungen gelten für arm und reich: Gesellschaftliche Fairness und ein Verzicht auf allzu große materielle und soziale Ungleichheiten sind für jedermann besser.

Solchen systemischen Zusammenhängen geht diese Ausgabe unserer Zeitschrift wieder in ihren vielfältigen Facetten quer durch die Lebensalter nach: Mit einem umfassenden und differenzierten Bericht zur Gesundheit von Jugendlichen in Deutschland, zur häuslichen Umgebung von Familien mit Kleinkindern als Setting für Präventionsmaßnahmen gegenüber Tabakrauch, zur Gesundheitsförderung bei älteren Menschen, zur Vergütung von Schwerverletzten im deutschen DRG-System, zu (Daten-) Qualitätsunterschieden in der onkologischen Versorgung, zum Zusammenhang von Preisen und Pflegequalität, zum Qualitätsmanagement und zur Infektionshygiene in der ambulanten Pflege sowie mit einem kurzen Bericht zu den Ergebnissen des EU-Projekts INTERLINKS zur Weiterentwicklung guter Langzeitpflege.

Deutschland: Armes reiches Land? In vieler Hinsicht wären die märchenerfahrenen Gebrüder Grimm auch in ihren politischen Ambitionen wohl sehr zufrieden mit dem, was seit 1848 auf deutschem Boden, wenn auch oft erst durch krisenhafte Übergänge, entstanden ist. Dabei tut ein kritischer Blick Not: Als Akt der Wachsamkeit gegenüber dem schon Erreichten, als ethisches Gebot und auch als Akt der Klugheit angesichts einer doch erheblichen Schere zwischen arm und reich, die weder den Armen noch den Reichen gut tut und in vielen Ausprägungen, damals wie heute, unverständlich ist. Auch wenn der Satz gilt: „Man ist reich, wenn es reicht“, bleibt bei zu großen Vermögensund Einkommensunterschieden eine gesellschaftliche Wunde zu diagnostizieren. Diese behindert Gemeinschaften in ihrer Entwicklung. Vielleicht ist angesichts des absolut gesehen großen Wohlstands in Europa die Zeit für einen Wertewandel am Kommen, für eine Hinwendung zu sogenannten postmateriellen Werten. Zeit für die neuen Stockwerke in der Maslowschen Bedürfnispyramide: Im Bereich des Sozialen wie bei der Selbstverwirklichung und den damit verbundenen Bedürfnissen nach Partizipation und Zugehörigkeit, nach Kreativität, Ästhetik, Kontemplation und Transzendenz [7]. Vielleicht unsere Chance, um „vergnügt bis an das Ende zu leben“.

\section{Literatur}

1 Maslow AH. Motivation and personality. Harper \& Row; New York: 1954 überarbeitete Ausgabe 1970

2 Maslow AH, Geiger H, Maslow BG. The Farther Reaches of Human Nature. Viking Press; New York: 1971

3 Deutscher Bundestag (Herausgeber). Lebenslagen in Deutschland - 1. Armuts- und Reichtumsbericht der Bundesregierung - Drucksache 14/5990. 2001

4 Bayerisches Landesamt für Gesundheit und Lebensmittelsicherheit. Erklärungsmodell regionaler Gesundheitsunterschiede. Erlangen LGL 2007 (s.a. http://www.lgl.bayern.de/gesundheit/gesundheitsbericht erstattung/index.htm)

5 Wilkinson R. Unhealthy Societes: The Affliction of Inequality. London: Cambridge; 1996

6 Wilkinson RG, Pickett $K$. The Spirit Level: Why More Equal Societies Almost Always Do Better. London, Allan Lane. 2009

7 Inglehart R. The Silent Revolution. Changing Values and Politic Styles among Western Publics. Princeton: Princeton University Press 1977 (dt. Übersetzung: Die stille Revolution. Vom Wandel der Werte. Königstein: Athenaeum 1982) 